\title{
Relation of blood pressure variability to left ventricular function and arterial stiffness in hypertensive patients
}

\author{
Sung-Hee $\underline{\text { Shin }}{ }^{1}$, MD, Ji-Hoon Jang ${ }^{1}$, Yong-Soo Baek ${ }^{1}$, Sung-Woo Kwon ${ }^{1}$, MD, Sang-Don Parkk ${ }^{1}$ MD, Seong-IIl Woo ${ }^{1}$, MD, \\ Dae-Hyeok Kim${ }^{1}, \mathrm{MD}$, Jun KWan ${ }^{1}, \mathrm{MD}$
}

INTRODUCTION Variability of blood pressure (BP) has been reported to be related to worse cardiovascular outcomes. We examined the impact of daytime systolic BP variability on left ventricular (LV) function and arterial stiffness in hypertensive patients.

METHODS Ambulatory BP monitoring (ABPM) and echocardiography were performed in 116 hypertensive patients. We assessed BP variability as standard deviations of daytime systolic BP on 24-hour ABPM. Conventional echocardiographic parameters, area strain and three-dimensional diastolic index (3D-DI) using 3D speckle tracking were measured. Arterial stiffness was evaluated by acquiring pulse wave velocity (PWV) and augmentation index.

RESULTS Patients with higher BP variability showed significantly increased left ventricular mass index (LVMI) and late mitral inflow velocity, as well as decreased E/A (early mitral inflow velocity/late mitral inflow velocity) ratio, area strain and 3D-DI than those with lower BP variability (LVMI: $p=0.02$; A velocity: $p<0.001$; E/A ratio: $p<0.001$; area strain: $p=0.02$; 3D-DI: $p=0.04)$. In addition, increased BP variability was associated with higher PWV and augmentation index $(p<0.001)$. Even among patients whose BP was well controlled, BP variability was related to LV mass, diastolic dysfunction and arterial stiffness.

CONCLUSION Increased BP variability was associated with LV mass and dysfunction, as well as arterial stiffness, suggesting that BP variability may be an important determinant of target organ damage in hypertensive patients.

Keywords: arterial stiffness, blood pressure, echocardiography

\section{INTRODUCTION}

Hypertension has been established to be a risk factor for cardiovascular events due to its effects on target organs. Many studies have indicated that blood pressure (BP) is predicative of cardiovascular morbidity and mortality. 24-hour ambulatory BP monitoring (ABPM) has been shown to provide a more representative estimate of $\mathrm{BP}$ level than conventional clinic BP and to be more closely related to target organ damage than office BP. ${ }^{(1-5)}$ In addition, for any given 24-hour average BP, target organ damage might be more pronounced in patients with greater $\mathrm{BP}$ variability. ${ }^{(6-10)}$ Therefore, in addition to BP itself, fluctuations in BP may also affect prognosis to some extent, as prior studies have reported. ${ }^{(1,2)}$

Daytime BP has higher levels and more fluctuation compared with nighttime BP. Systolic BP variability in the daytime has also been reported to be predictive of cardiovascular outcome. ${ }^{(11,12)}$ Hence, we examined if daytime systolic BP variability impacts left ventricular (LV) structure and function as well as arterial stiffness in hypertensive patients.

\section{METHODS}

Consecutive hypertensive patients were enrolled from Inha University Hospital, South Korea. All procedures performed in this study were in accordance with the ethical standards of the institutional research committee and with the 1964 Helsinki Declaration and its later amendments or comparable ethical standards. We excluded patients who had atrial fibrillation or significant arrhythmia, LV ejection fraction $<50 \%$, regional wall motion abnormality or significant valvular disease. ABPM, echocardiography and measurements of arterial stiffness were performed, and the relationship among BP variability, echocardiographic parameters and arterial stiffness was assessed.

ABPM results were recorded over 24 hours during the patient's normal daily activities with an autonomic device (ABD-Monitor 90207; Spacelabs, Snoqualmie, WA, USA) that was programmed to obtain the readings at a 30-minute interval during daytime (defined as between 6 am and $12 \mathrm{am}$ ) and at a one-hour interval during nighttime (defined as between 12 am and $6 \mathrm{am})$. The following readings were omitted due to the potential presence of technical artefacts: systolic BP $>250 \mathrm{mmHg}$ or $<70 \mathrm{mmHg}$, diastolic BP > $200 \mathrm{mmHg}$ or $<40 \mathrm{mmHg}$, or pulse pressure $>200 \mathrm{mmHg}$ or $<20 \mathrm{mmHg}$. BP variability was assessed as standard deviations (SDs) of daytime systolic BP (SBP) and diastolic BP (DBP) on the 24-hour ABPM. Nocturnal dipping was defined as a decline in average SBP or DBP at nighttime of more than $10 \%$ compared with average daytime BP. ${ }^{(13)}$ Pulse pressure variability was calculated as SDs of pulse pressure on the ABPM.

Imaging was performed with a commercially available echocardiographic system (Artida ${ }^{\mathrm{TM}}$; Toshiba, Tokyo, Japan). Conventional parameters were measured, including peak early (E) and late $(\mathrm{A})$ mitral inflow velocity, the E/A ratio, deceleration time of early mitral flow velocity (DT) and early mitral annular

${ }_{1}^{1}$ Division of Cardiology, Inha University College of Medicine, Incheon, South Korea

Correspondence: Dr Sung-Hee Shin, Associate Professor, Division of Cardiology, Department of Internal Medicine, Inha University College of Medicine, 7-206, 3-GA, Shinheung-Dong, Jung-Gu, Incheon, 400-711, South Korea. sshin@inha.ac.kr 
velocity (Em) at the septal annular site. LV internal dimension (LVID), septal wall thickness (SWT) and posterior wall thickness (PWT) were measured at end-diastole according to American Society of Echocardiography recommendations. ${ }^{(14)}$ LV mass (in grams) was calculated with the following formula and corrected for body surface: $L V$ mass $=0.8\left\{1.04 \times\left[(\text { LVID }+ \text { PWT }+ \text { SWT })^{3}\right.\right.$ $\left.\left.-(\text { LVID })^{3}\right]\right\}+0.6$.

LV hypertrophy $(\mathrm{LVH})$ was defined as LV mass index $>95 \mathrm{~g} / \mathrm{m}^{2}$ for women and $>115 \mathrm{~g} / \mathrm{m}^{2}$ for men. ${ }^{(14)}$ Relative wall thickness (RWT) was calculated with the formula (PWT + SWT)/ LVID. In addition, three-dimensional (3D) full-volume images were acquired with a volume rate of 20-30 volumes/second, and recently developed 3D speckle tracking was applied to the $3 \mathrm{D}$ volume dataset to assess the global area strain (Artida; Toshiba, Tokyo, Japan). ${ }^{(15,16)}$ 3D wall motion-tracking software automatically showed 3D data with apical two-chamber, four-chamber and three short-axis views at different levels of the LV (base, middle and apex). After adjusting the orientation of two-dimensional (2D) planes and tracing LV endocardial and epicardial borders on long-axis images manually, the 3D endocardial contour was reconstructed and tracked in 3D data from frame to frame throughout the cardiac cycle, following which 3D strain data was displayed. Tracking quality was verified for each segment with manual adjustment if needed. 3D diastolic index of area strain (3D-DI) was calculated as percentage change of global area strain during the first one-third of the diastolic period. ${ }^{(17,18)}$ Global area strain and 3D-DI were averaged from 16 LV segments.

Arterial stiffness was evaluated by acquiring pulse wave velocity (PWV) and augmentation index (AIx) with radial artery applanation tonometry (SphygmoCor ${ }^{\circledR}$; AtCor Medical, New South Wales, Australia). The patients refrained from smoking, heavy meals, drinking alcohol or caffeinated beverages, and antihypertensive medication on the day of examination. A small pencil-sized tonometer was placed on the skin over the carotid and femoral arteries, and steady pulse waveforms were obtained on the sites. Carotid-femoral PWV was determined from measurements of pulse transit time and the distance travelled between the two recording sites using cardiac gating to the $\mathrm{R}$ wave in electrocardiography. Alx, the difference between early and late pressure peaks divided by pulse pressure, was also calculated with a computer algorithm. Alx was adjusted to a standard heart rate of 75 beats/minute, based on previous studies showing that it was influenced by heart rate. ${ }^{(19)}$

Summarised data for continuous variables was expressed as mean $\pm S D$, while categorical variables were expressed as number and percentage. We categorised the patients in tertiles according to the SD of the mean of daytime BP or pulse pressure variability and applied a trend test, an extension of the Wilcoxon rank-sum tests, for assessing a systemic increase or decrease across the ordered groups. Multiple regression analysis was used to control for the effects of SBP on the relationship between the measurements and daytime SBP variability. A p-value $<0.05$ was considered as statistically significant.

\section{RESULTS}

A total of 116 consecutive hypertensive patients were enrolled (age $57 \pm 12$ years, 56 male, 60 female) and categorised into three tertiles according to daytime SBP variability. Daytime SBP variability was associated with older age $(p<0.001)$ and female gender ( $p=0.02$; Table I). The prevalence of diabetes mellitus $(p=0.72)$ and dyslipidaemia $(p=0.06)$ was similar among the groups. For medication, the patients with greater BP variability were more likely to take beta blockers than those with less BP variability $(p=0.01)$.

Table II shows BP results and their variability in patients in the three tertiles. SBP was significantly higher in patients who were in the uppermost tertile of daytime SBP variability $(p<0.001)$, whereas DBP was not significantly different among the groups $(p=0.89)$. Nocturnal decreases in SBP and DBP were not significant across the three groups.

Patients with higher daytime SBP variability showed significantly increased LV mass $(p=0.02)$ and A velocity $(p<0.001)$, and decreased E/A ratio $(p<0.001)$, Em $(p=0.02)$, area strain $(p=0.02)$ and $3 \mathrm{D}-\mathrm{DI}(\mathrm{p}=0.04)$ than those with lower $B P$ variability (Table III). In addition, increased BP variability was associated with higher PWV $(p<0.001)$ and AIx $(p<0.001)$. Such relationships between BP variability and echocardiographic or arterial stiffness parameters were not apparent for nighttime SBP variability as well as daytime or nighttime DBP variability. Even among 68 patients whose BP was well controlled $(<140 / 90$ $\mathrm{mmHg}$ ), BP variability was related to LV mass, diastolic dysfunction and arterial stiffness (LV mass: $p=0.03$; A velocity: $p<0.001$, E/A ratio: $p=0.02$; PWV: $p=0.04$; Alx: $p<0.001$ ). When adjusted for SBP in the whole cohort, the relationships between variables remained similar (RWT: $p=0.005$; A velocity: $p<0.001$; E/A ratio: $p=0.01$; area strain: $p=0.04$; PWV: $\mathrm{p}=0.009 ; \mathrm{Alx}: \mathrm{p}<0.001)$.

$41(35 \%)$ patients showed the presence of $\mathrm{LVH}$. In patients without LVH, SBP variability was associated with LV diastolic function and arterial stiffness (A velocity: $p=0.03$; PWV: $p=0.008$; Alx: $p<0.001)$. On the other hand, SBP variability in patients with LVH had a significant relationship with LV diastolic function but not with arterial stiffness (E velocity: $p=0.001$; A velocity: $p=0.02$; E/A ratio: $p<0.001 ; D T: p<0.001 ; E m: p=0.002$ ). Patients with LVH had higher values of arterial stiffness than those without LVH (PWV: $p=0.03$; Alx: $p=0.003$ ). In addition, patients with higher pulse pressure variability showed increased LV mass, poorer diastolic function and higher arterial stiffness (LV mass: $p=0.018$; RWT: $p=0.001 ;$ A velocity: $p<0.001 ; E / A$ ratio: $p=0.001 ; E m:$ $p=0.006 ; E / E m: p=0.04 ;$ PWV: $p=0.001 ; A l x: p=0.01$ ).

\section{DISCUSSION}

Our study showed that older age, female gender and beta-blocker use were associated with higher daytime SBP variability in the hypertensive patients. In addition, daytime SBP variability was related to LV mass, LV diastolic function and arterial stiffness even in the patients whose BP was well controlled, which might suggest BP variability can be attributable to vascular and myocardial damage. These findings are compatible with 
Table I. Baseline demographic variables according to daytime SBP variability.

\begin{tabular}{|lllll|}
\hline Parameter & \multicolumn{3}{c}{ No. $(\%) /$ mean \pm SD } & p-value \\
\cline { 2 - 4 } & $<\mathbf{1 2}(\mathbf{n}=\mathbf{3 9 )}$ & $\mathbf{1 2 - 1 5}(\mathbf{n}=\mathbf{3 9})$ & $\mathbf{1 0}(\mathbf{n}=\mathbf{3 8})$ & $<0.001$ \\
\hline Age $(\mathbf{y r})$ & $51.0 \pm 9.3$ & $57.8 \pm 12.0$ & $61.1 \pm 12.7$ & 0.02 \\
\hline Female gender & $13(33)$ & $24(62)$ & $23(61)$ & 0.72 \\
\hline Diabetes mellitus & $4(10)$ & $3(8)$ & $2(5)$ & 0.06 \\
\hline Dyslipidaemia & $8(21)$ & $17(44)$ & $16(42)$ & 0.75 \\
\hline Medication & & & $7(18)$ & 0.11 \\
\hline Aspirin & $7(18)$ & $5(13)$ & $19(50)$ & 0.01 \\
\hline ACE inhibitor/ARB & $26(67)$ & $18(46)$ & $8(21)$ & 0.09 \\
\hline Beta blocker & $1(3)$ & $5(13)$ & $15(39)$ & 0.95 \\
\hline Calcium channel blocker & $21(54)$ & $25(64)$ & $12(32)$ & 0.32 \\
\hline Diuretic & $11(28)$ & $11(28)$ & $5(13)$ & \\
\hline Statin & $4(10)$ & $4(10)$ & & \\
\hline
\end{tabular}

ACE: angiotensin-converting enzyme; ARB: angiotensin receptor blocker; SD: standard deviation

Table II. Ambulatory blood pressure parameters according to daytime SBP variability.

\begin{tabular}{|c|c|c|c|c|}
\hline \multirow[t]{2}{*}{ Parameter } & \multicolumn{3}{|c|}{ No. $(\%) /$ mean \pm SD } & \multirow[t]{2}{*}{ p-value } \\
\hline & $<12(n=39)$ & $12-15(n=39)$ & $\geq 15(n=38)$ & \\
\hline SBP (mmHg) & $129 \pm 12$ & $131 \pm 10$ & $140 \pm 13$ & $<0.001$ \\
\hline DBP (mmHg) & $84 \pm 7$ & $84 \pm 9$ & $85 \pm 12$ & 0.89 \\
\hline SBP variability $(\mathrm{mmHg})$ & $10.6 \pm 2.1$ & $13.8 \pm 1.5$ & $19.2 \pm 4.1$ & $<0.001$ \\
\hline DBP variability (mmHg) & $9.0 \pm 1.8$ & $10.1 \pm 1.7$ & $12.0 \pm 2.7$ & $<0.001$ \\
\hline \multicolumn{5}{|l|}{ Dippers } \\
\hline SBP & $17(44)$ & $15(38)$ & $23(61)$ & 0.09 \\
\hline DBP & $23(59)$ & $16(41)$ & $16(42)$ & 0.12 \\
\hline
\end{tabular}

DBP: diastolic blood pressure; SBP: systolic blood pressure; SD: standard deviation

prior studies demonstrating that SBP variability was related to subclinical target organ damage. ${ }^{(8,20-23)}$ Additionally, when we assessed pulse pressure variability, it was also associated with LV diastolic function and arterial stiffness.

$\mathrm{BP}$ variability increases along with sympathetic activity and can be enhanced in the hypertensive population. It is influenced by central, humoral, reflex and behavioural factors as well as the sympathetic nervous system. ${ }^{(24,25)}$ The central autonomic drive, mediated by the baroreflex mechanism, may contribute to BP variability. In previous studies, BP variability was reported to be inversely correlated with the sensitivity of the baroreceptorheart rate reflex or BP reflex, as assessed using vasoactive drug technique in hypertensive subjects, suggesting that baroreflex regulation may be a potential determinant of BP variability. ${ }^{(26,27)}$ In animal studies, sinoaortic denervation resulted in markedly increased BP variability. ${ }^{(28)}$ Considering these findings, impaired arterial baroreflex, which is related to increased arterial stiffness, could be partly responsible for BP variability. In our study, PWV and Alx were associated with greater BP variability, while patients with older age and female gender relating to increased arterial stiffness had higher BP variability. Interestingly, in our study, SBP variability was significantly related to LV diastolic function and arterial stiffness in patients without LVH, whereas in those with $L V H$, the only significant relationship was with LV diastolic function. This might be a result of less statistical power due to the small number of the study population. However, it might also suggest that SBP variability affects arterial stiffness more in patients without LVH. In hypertensive patients who have already developed LVH, SBP variability can still be related to worsened LV diastolic function, but it might affect arterial stiffness less significantly, since their arteries have already stiffened.

We herein utilised newly developed 3D speckle tracking to assess the impact of SBP variability on subclinical systolic function. Strain has been reported to enable early detection of subtle changes in cardiac function, while 3D speckle tracking was recently developed to overcome the inherent drawbacks of 2D speckle tracking, such as the use of foreshortened images and motion of the speckles in and out of the imaging plane. Area strain, a new parameter based on the 3D speckle tracking method, refers to a percentile change of area in the endocardial layer and represents a combined parameter for longitudinal and circumferential strain. ${ }^{(14,15,18,29,30)}$ Several studies suggested that area strain was more sensitive to the changes of regional deformation and produces more reproducible results than other unidimensional deformation parameters, including longitudinal or circumferential strains. ${ }^{(31-33)}$ It may reduce tracking error through combining two types of directional deformation data and increasing the signal-to-noise ratio. In our data, the patients with higher SBP variability had lower area strain than those with lower SBP variability, suggesting that SBP variability might be related 
Table III. Echocardiographic characteristics and arterial stiffness according to daytime SBP variability.

\begin{tabular}{|c|c|c|c|c|}
\hline \multirow[t]{2}{*}{ Parameter } & \multicolumn{3}{|c|}{ Mean \pm SD } & \multirow[t]{2}{*}{ p-value } \\
\hline & $<12(n=39)$ & $12-15(n=39)$ & $\geq 15(n=38)$ & \\
\hline \multicolumn{5}{|l|}{ Conventional parameter } \\
\hline $\operatorname{LVEDD~}(\mathrm{mm})$ & $46.4 \pm 3.4$ & $46.0 \pm 3.5$ & $46.3 \pm 4.2$ & 0.40 \\
\hline LVESD (mm) & $31.0 \pm 2.6$ & $30.2 \pm 3.4$ & $30.0 \pm 3.5$ & 0.08 \\
\hline LVEF (\%) & $62.5 \pm 4.2$ & $63.1 \pm 3.9$ & $62.2 \pm 5.2$ & 0.74 \\
\hline LVMI $\left(\mathrm{g} / \mathrm{m}^{2}\right)$ & $93.7 \pm 19.6$ & $94.6 \pm 16.2$ & $103.6 \pm 21.2$ & 0.02 \\
\hline Relative wall thickness & $0.41 \pm 0.05$ & $0.44 \pm 0.05$ & $0.46 \pm 0.07$ & 0.05 \\
\hline LA dimension (mm) & $37.4 \pm 4.0$ & $37.8 \pm 4.5$ & $38.4 \pm 5.1$ & 0.32 \\
\hline E velocity $(\mathrm{cm} / \mathrm{s})$ & $66.5 \pm 16.5$ & $67.3 \pm 12.7$ & $65.1 \pm 15.1$ & 0.68 \\
\hline A velocity $(\mathrm{cm} / \mathrm{s})$ & $66.6 \pm 13.7$ & $80.2 \pm 17.4$ & $81.4 \pm 19.6$ & $<0.001$ \\
\hline E/A ratio & $1.03 \pm 0.32$ & $0.88 \pm 0.27$ & $0.83 \pm 0.22$ & $<0.001$ \\
\hline Deceleration time (ms) & $248.9 \pm 49.8$ & $247.0 \pm 53.5$ & $263.5 \pm 45.0$ & 0.10 \\
\hline $\mathrm{Em}(\mathrm{cm} / \mathrm{s})$ & $8.3 \pm 1.3$ & $7.4 \pm 1.9$ & $7.2 \pm 1.9$ & 0.02 \\
\hline $\mathrm{E} / \mathrm{Em}$ & $8.6 \pm 2.3$ & $9.6 \pm 2.6$ & $9.6 \pm 2.7$ & 0.24 \\
\hline \multicolumn{5}{|l|}{ 3D STE } \\
\hline Area strain (\%) & $41.8 \pm 4.2$ & $40.3 \pm 3.8$ & $38.4 \pm 4.1$ & 0.02 \\
\hline 3D diastolic index (\%) & $35.1 \pm 12.3$ & $27.2 \pm 10.8$ & $26.8 \pm 12.6$ & 0.04 \\
\hline \multicolumn{5}{|l|}{ Arterial stiffness } \\
\hline Pulse wave velocity (m/s) & $8.7 \pm 1.1$ & $9.6 \pm 2.1$ & $10.3 \pm 2.4$ & $<0.001$ \\
\hline Augmentation index (\%) & $21.1 \pm 9.8$ & $28.9 \pm 9.7$ & $31.0 \pm 5.9$ & $<0.001$ \\
\hline
\end{tabular}

A: late mitral inflow; E: peak early mitral inflow; Em: early mitral annular velocity; LA: left atrium; LVEDD: left ventricular end-diastolic dimension; LVESD: left ventricular end-systolic dimension; LVEF: left ventricular ejection fraction; LVMI: left ventricular mass index; SBP: systolic blood pressure; SD: standard deviation; STE: speckle tracking echocardiography

to LV subtle systolic dysfunction. In the group with increased SBP variability, LV was also more hypertrophied, and diastolic function, assessed using conventional Doppler echocardiography and 3D speckle tracking, was more impaired. BP variation may cause traumatic stress on the vessel wall, thus promoting early target organ damage. ${ }^{(34)}$

In the present study, the patients with higher BP variability more frequently received beta blockers. Even though the number was small, making a causal relationship uncertain, some beta blockers might have a worsening impact on BP variability. Decreasing BP variability may provide additive benefits to BP control in protecting against target organ damage to some extent. Similarly, antihypertensive drugs that reduce SBP variation in addition to average SBP may be more beneficial for hypertensive patients rather than simply lowering average SBP. Further studies are necessary to address the effect of antihypertensive drugs on $B P$ variability and their possible differential impact on prognosis.

In our study, we used daytime SBP variability and not 24-hour $\mathrm{BP}$ variability to assess BP variability. 24-hour BP variability can be affected by BP decline between day and night, or dipping, which is reported to have a protective effect on target organ damage. ${ }^{(35,36)}$ To exclude this possible confounding factor, we used daytime SBP variability and not overall 24-hour BP variability in assessing BP variation. In addition, prior studies have reported that daytime SBP variability was predictive of cardiovascular mortality and morbidity even after adjusting risk factors attributable to BP elevation. ${ }^{(11,12)}$

Our study was limited by the relatively small population size. Also, our BP devices were set to record with a measurement frequency set at 30-minute intervals at daytime and 60 minutes at nighttime. More frequent measurements might be better for more accurate and reliable assessment of BP variability. In addition, since our study is cross-sectional, its findings cannot show a causal relationship between BP variability and LV mass or arterial stiffness. However, prior animal studies revealing cardiac damage and aortic hypertrophy in sinoaortic-denervated rats, an experimental model of high BP variability without hypertension, suggest that BP fluctuations might alter cardiac morphology and arterial stiffness. ${ }^{(37)}$ Wide swings in BP may play a role in the development of endothelial dysfunction and atherosclerosis, thus eventually leading to worse cardiovascular outcomes. ${ }^{(12,38,39)}$

In conclusion, increased BP variability was associated with LV mass and dysfunction as well as arterial stiffness. This suggests that BP variability was one of the important determinants of cardiac and vascular target organ damage in the hypertensive patients, although we could not determine the causality between BP variability and organ damage because of the study's crosssectional design.

\section{ACKNOWLEDGEMENT}

This work was supported by the Inha University Research Grant (INHA-59279).

\section{REFERENCES}

1. Clement DL, De Buyzere ML, De Bacquer DA, et al; Office versus Ambulatory Pressure Study Investigators. Prognostic value of ambulatory blood-pressure recordings in patients with treated hypertension. N Engl J Med 2003; 348:2407-15. 
2. Khattar RS, Swales JD, Dore C, Senior R, Lahiri A. Effect of aging on the prognostic significance of ambulatory systolic, diastolic, and pulse pressure in essential hypertension. Circulation 2001; 104:783-9.

3. Perloff D, Sokolow M, Cowan R. The prognostic value of ambulatory blood pressures. JAMA 1983; 249:2792-8.

4. Redon J, Campos C, Narciso ML, et al. Prognostic value of ambulatory blood pressure monitoring in refractory hypertension: a prospective study. Hypertension 1998; 31:712-8.

5. Khattar RS, Swales JD, Banfield A, et al. Prediction of coronary and cerebrovascular morbidity and mortality by direct continuous ambulatory blood pressure monitoring in essential hypertension. Circulation 1999; 100:1071-6.

6. Mancia G, Parati G, Hennig M, et al; ELSA Investigators. Relation between blood pressure variability and carotid artery damage in hypertension: baseline data from the European Lacidipine Study on Atherosclerosis (ELSA). J Hypertens 2001; 19:1981-9.

7. Frattola A, Parati G, Cuspidi C, Albini F, Mancia G. Prognostic value of 24-hour blood pressure variability. J Hypertens 1993; 11:1133-7.

8. Tatasciore A, Renda G, Zimarino M, et al. Awake systolic blood pressure variability correlates with target-organ damage in hypertensive subjects. Hypertension 2007; 50:325-32

9. Muntner $\mathrm{P}$, Shimbo D, Tonelli $\mathrm{M}$, et al. The relationship between visit-to-visit variability in systolic blood pressure and all-cause mortality in the genera population: findings from NHANES III, 1988 to 1994. Hypertension 2011 57:160-6.

10. Rothwell PM, Howard SC, Dolan E, et al. Prognostic significance of visit-to-visi variability, maximum systolic blood pressure, and episodic hypertension. Lance 2010; 375:895-905

11. Kikuya M, Hozawa A, Ohokubo T, et al. Prognostic significance of blood pressure and heart rate variabilities: the Ohasama study. Hypertension 2000; 36:901-6

12. Sander D, Kukla C, Klingelhöfer J, Winbeck K, Conrad B. Relationship between circadian blood pressure patterns and progression of early carotid atherosclerosis: a 3-year follow-up study. Circulation 2000; 102:1536-41.

13. Verdecchia P, Schillaci G, Guerrieri M, et al. Circadian blood pressure change and left ventricular hypertrophy in essential hypertension. Circulation 1990 81:528-36.

14. Lang RM, Bierig M, Devereux RB, et al; Chamber Quantification Writing Group American Society of Echocardiography's Guidelines and Standards Committee European Association of Echocardiography. Recommendations for chambe quantification: a report from the American Society of Echocardiography's Guidelines and Standards Committee and the Chamber Quantification Writing Group, developed in conjunction with the European Association of Echocardiography, a branch of the European Society of Cardiology. J Am Soc Echocardiogr 2005; 18:1440-63.

15. Urbano-Moral JA, Patel AR, Maron MS, Arias-Godinez JA, Pandian NG. Threedimensional speckle-tracking echocardiography: methodological aspects and clinical potential. Echocardiography 2012; 29:997-1010.

16. Mor-Avi V, Lang RM, Badano LP, et al. Current and evolving echocardiographic techniques for the quantitative evaluation of cardiac mechanics: ASE/EAE consensus statement on methodology and indications endorsed by the Japanese Society of Echocardiography. J Am Soc Echocardiogr 2011; 24:277-313.

17. Ishii K, Suyama T, Imai M, et al. Abnormal regional left ventricular systolic and diastolic function in patients with coronary artery disease undergoing percutaneous coronary intervention: clinical significance of post-ischemic diastolic stunning. J Am Coll Cardiol 2009; 54:1589-97.

18. Shin SH, Park SD, Woo SI, et al. Utility of global strain by two-dimensional and three-dimensional speckle tracking for assessing left ventricular diastolic function: comparison with pressure wire analysis. Korean Circ J 2013 43:615-21.
19. Wilkinson IB, MacCallum $\mathrm{H}$, Flint $\mathrm{L}$, et al. The influence of heart rate on augmentation index and central arterial pressure in humans. J Physiol 2000; 525 Pt 1:263-70.

20. Ozawa M, Tamura K, Okano Y, et al. Blood pressure variability as well as blood pressure level is important for left ventricular hypertrophy and brachial-ankle pulse wave velocity in hypertensives. Clin Exp Hypertens 2009; 31:669-79.

21. Kotsis V, Stabouli S, Karafillis I, et al. Arterial stiffness and $24 \mathrm{~h}$ ambulatory blood pressure monitoring in young healthy volunteers: the early vascular ageing Aristotle University Thessaloniki Study (EVA-ARIS Study). Atherosclerosis 2011; 219:194-9.

22. Lee HT, Lim YH, Kim BK, et al. The relationship between ambulatory arterial stiffness index and blood pressure variability in hypertensive patients. Korean Circ J 2011; 41:235-40

23. Chi C, Yu SK, Auckle R, et al. Association of left ventricular structural and functional abnormalities with aortic and brachial blood pressure variability in hypertensive patients: the SAFAR study. J Hum Hypertens 2017; 31:633-9.

24. Conway J, Boon N, Davies C, Jones JV, Sleight P. Neural and humoral mechanisms involved in blood pressure variability. J Hypertens 1984; 2:203-8.

25. Mancia G, Grassi G. Mechanisms and clinical implications of blood pressure variability. J Cardiovasc Pharmacol 2000; 35(7 Suppl 4):S15-9.

26. Cowley AW Jr, Liard JF, Guyton AC. Role of baroreceptor reflex in daily control of arterial blood pressure and other variables in dogs. Circ Res 1973; 32:564-76.

27. Mancia G, Parati G, Pomidossi G, et al. Arterial baroreflexes and blood pressure and heart rate variabilities in humans. Hypertension 1986; 8:147-53.

28. Ramirez AJ, Bertinieri G, Belli L, et al. Reflex control of blood pressure and heart rate by arterial baroreceptors and by cardiopulmonary receptors in the unanaesthetized cat. J Hypertens 1985; 3:327-35.

29. Marwick TH. Measurement of strain and strain rate by echocardiography: ready for prime time? J Am Coll Cardiol 2006; 47:1313-27

30. Gorcsan J 3rd, Tanaka H. Echocardiographic assessment of myocardial strain. J Am Coll Cardiol 2011; 58:1401-13.

31. Wen H, Liang Z, Zhao Y, Yang K. Feasibility of detecting early left ventricular systolic dysfunction using global area strain: a novel index derived from threedimensional speckle-tracking echocardiography. Eur J Echocardiogr 2011; 12:910-6.

32. Shin SH, Suh YJ, Baek YS, et al. Impact of area strain by 3D speckle tracking on clinical outcome in patients after acute myocardial infarction. Echocardiography 2016; 33:1854-9.

33. Seo Y, Ishizu T, Aonuma K. Current status of 3-dimensional speckle tracking echocardiography: a review from our experiences. J Cardiovasc Ultrasound $2014 ; 22: 49-57$.

34. Veerabhadrappa P, Diaz KM, Feairheller DL, et al. Enhanced blood pressure variability in a high cardiovascular risk group of African Americans: FIT4Life Study. J Am Soc Hypertens 2010; 4:187-95.

35. Staessen JA, Bieniaszewski L, O'Brien E, et al. Nocturnal blood pressure fall on ambulatory monitoring in a large international database. The 'Ad Hoc' Working Group. Hypertension 1997; 29(1 Pt 1):30-9.

36. Izzedine H, Launay-Vacher V, Deray G. Abnormal blood pressure circadian rhythm: a target organ damage? Int J Cardiol 2006; 107:343-9.

37. Miao CY, Xie HH, Zhan LS, Su DF. Blood pressure variability is more important than blood pressure level in determination of end-organ damage in rats. J Hypertens 2006; 24:1125-35.

38. Mancia G, Parati G, Castiglioni P, et al. Daily life blood pressure changes are steeper in hypertensive than in normotensive subjects. Hypertension 2003; 42:277-82.

39. Kario K. Systemic hemodynamic atherothrombotic syndrome and resonance hypothesis of blood pressure variability: triggering cardiovascular events. Korean Circ J 2016; 46:456-67. 